\title{
Der Nerventester
}

PETER BROCHWICZ Nerven sind dynamisch und sensibel. Doch manchmal

können sie auch nerven - wie Peter Brochwicz in seiner Bachelorarbeit feststellen musste. Um zu untersuchen, wie weit zervikale Mobilisationen den Nervus

medianus beeinflussen, lernte er die Kunst der Sonografie und der Geduld.

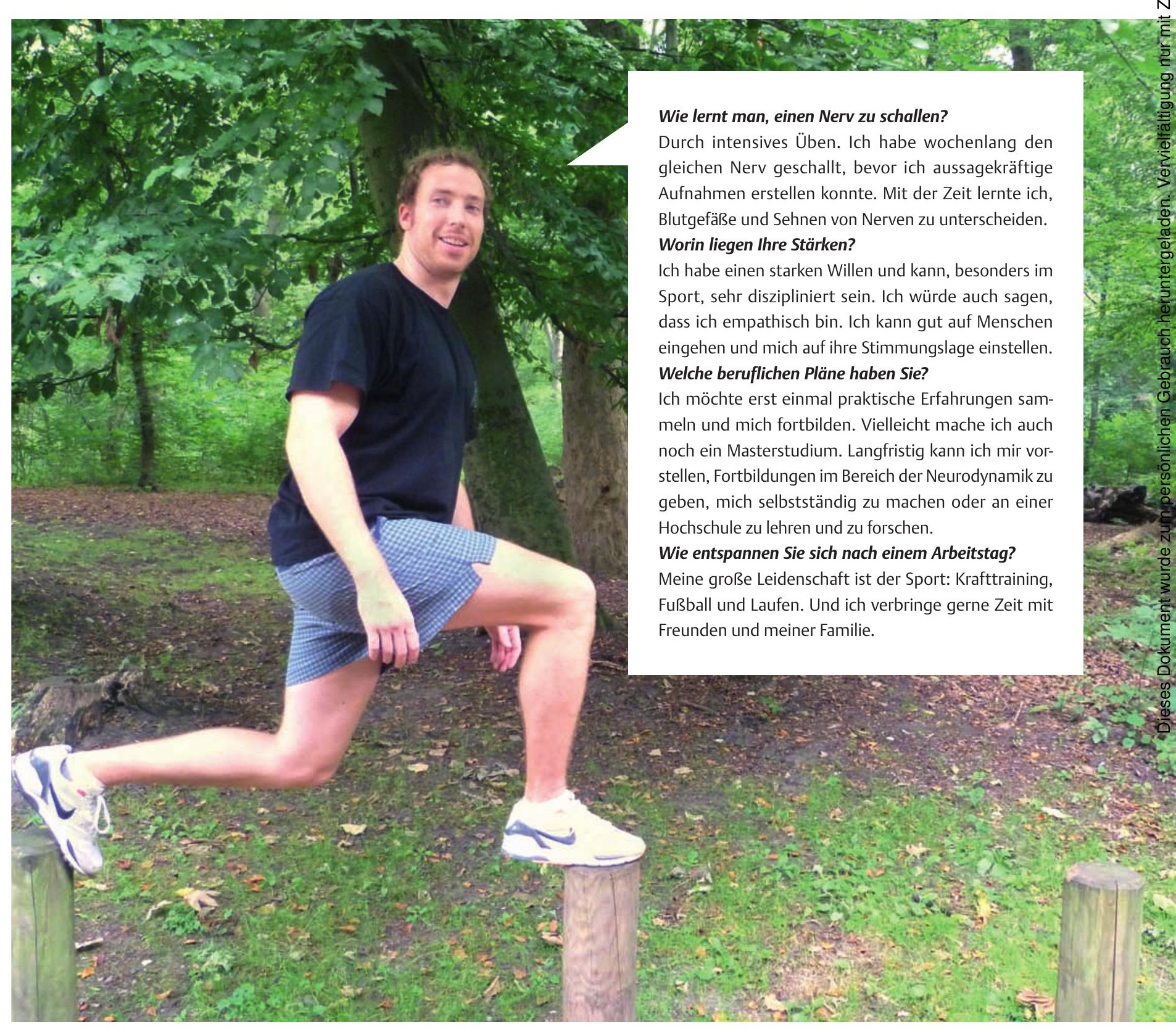




\section{Das Bewegungsverhalten des Nervus medianus bei Mobilisation der HWS}

\section{Peter Brochwicz ...}

... ist 27 Jahre alt und lebt in Hannover. Seit 2010 arbeitet er im Therapiezentrum Langenhagen mit Schwerpunkt auf Manueller Therapie. Direkt im Anschluss an seine Ausbildung zum Physiotherapeuten absolvierte er den Studiengang Physiotherapie an der Hochschule Osnabrück. Im Laufe der Zeit entwickelte er ein besonderes Interesse an der Neurodynamik. Dieses führte ihn auch zum Thema seiner Bachelorarbeit. Doch die gestaltete sich recht hürdenreich: Peter Brochwicz hatte vor seiner Studie nie mit diagnostischem Ultraschall gearbeitet. Dies hatte zur Folge, dass auf den ersten Aufnahmen alles Mögliche zu sehen war, nur nicht der gesuchte Nervus medianus. Nachdem er sein diagnostisches Geschick durch wochenlanges Üben endlich verbessert hatte, streikte die in England entwickelte Software zur Auswertung der Aufnahmen. Wieder war er in seiner Forschung unterbrochen und um Tage zurückgeworfen. Peter Brochwicz lernte also bei seiner Studie vor allem eins: durchhalten. Belohnt wurde er dafür mit vielen neuen Erkenntnissen über sein Interessengebiet und dem ersten Platz beim IFK-Wissenschaftspreis 2010.

\section{Die Bachelorarbeit}

Seit den 70er Jahren wurden verschiedene Techniken entwickelt, um das Nervengewebe zu untersuchen und zu mobilisieren. Eine davon ist das Cervical Lateral Glide (CLG), bei der Therapeuten eine kontralaterale Translationsbewegung der HWS hauptsächlich auf Höhe des Segments C 5/ C 6 durchführen. Sie wirkt unter anderem schmerzlindernd.

Peter Brochwicz untersuchte in seiner Studie, inwieweit das CLG im Vergleich zu einer kontralateralen Seitneigung der Halswirbelsäule speziell den Nervus medianus beeinflusst. Ihn interessierte, ob und wie weit sich der Nervus medianus bei diesen Bewegungen peripher mitbewegt. Zusätzlich untersuchte Peter Brochwicz, ob sich die Bewegung des Nervus medianus bei einem Karpaltunnelsyndrom (CTS) verändert. Diese Erkenntnis könnte zum Beispiel für die Behandlung des CTS von Nutzen sein.

In seine Studie bezog er 19 Frauen und 8 Männer ein. Um bei den allesamt gesunden Probanden neuronale Störungen auszuschließen, setzte er zwei Untersuchungsverfahren ein: den DASH-Fragebogen (Disability of Arm, Shoulder and Hand), der die Behinderungen im Alltag durch Schulter-, Arm- und Handprobleme erfasst, und den ULNT1 (Upper Limb Neurodynamic Test 1), der Auskunft über die Dynamik und Irritierbarkeit des Nervus medianus gibt. Indem Peter Brochwicz zudem zwei Frauen mit einem Karpaltunnelsyndrom untersuchte, erhielt er einen ersten Eindruck darüber, wie sich die Armnerven bei einer Pathologie verhalten. Um die Bewegungen des Nervs darzustellen und zu messen, nutzte er den diagnostischen Ultraschall. Dieser gilt in der Forschung als effektives Instrument zur Darstellung von Nerven. Er selbst schallte, während ein Kommilitone das laterale Gleiten durchführte. Dabei mobilisierte dieser die HWS in einem Rhythmus von zwei bis drei Sekunden bis an die Spannungsgrenze des
Gewebes. Peter Brochwicz maß die transversalen Bewegungen des Mediannervs an der Mitte des rechten Ober- und Unterarms sowie am Handgelenk. Die Bewegungen des Nervs in longitudinaler Richtung ermittelte er am mittleren und distalen Unterarm. Er wertete die Aufnahmen direkt am Ultraschallgerät und mit Hilfe einer speziellen Software aus.

\section{Ergebnisse}

Peter Brochwicz hat herausgefunden, dass...

> das CLG und die Lateralflexion zur Gegenseite das Bewegungsverhalten des Nervus medianus in transversaler und longitudinaler Richtung beeinflussen.

> bei beiden zervikalen Bewegungsmanövern die Nervenbewegungen bis ins Handgelenk weiterlaufen.

> das CLG den Nervus medianus signifikant stärker bewegt als die Lateralflexion.

$>$ die transversale Nervenbewegung bei den zwei Frauen mit CTS an Oberarm und Handgelenk signifikant vermindert war.

\section{Fazit}

Zusammenfassend kann Peter Brochwicz festhalten, dass...

> er neue Erkenntnisse über das Bewegungsverhalten des Nervus medianus während zervikaler Bewegungen gewinnen konnte.

> es sinnvoll wäre, die Erkenntnisse in einer Vergleichsstudie mit einer großen Anzahl gesunder und an CTS leidender Probanden zu überprüfen. Dabei wäre interessant, neben den Bewegungsrichtungen des Nervus medianus auch seine Spannungsveränderung zu untersuchen.

Eva Trompetter

$\Rightarrow$ Brochwicz P. Bewegungsverhalten des Nervus medianus bei zervikalen Bewegungsmanövern mittels sonografischer Diagnostik. Bachelorarbeit an der Fachhochschule Osnabrück; 2010 